\title{
Heat and groundwater transport between the Antarctic Ice Sheet and subglacial sedimentary basins from electromagnetic geophysical measurements
}

\author{
Bernd Kulessa*, Swansea University, UK; Kerry Key, Columbia University, USA; Sarah Thompson, University \\ of Tasmania, Australia; Martin Siegert, Imperial College London, UK
}

\section{Summary}

Numerical models of contemporary as well as paleo-ice sheets suggest that groundwater and heat exchanges between subglacial sedimentary basins and the ice sheet above, can be substantial and influence the flow of ice above. So far, an approach for the measurement and assessment of such heat fluxes has not been available. Here, we summarise existing evidence for groundwater and heat exchanges between contemporary and paleo ice sheets and the substrate below. We then explain the utility of electromagnetic geophysical measurements in elucidating such exchanges, and present magnetotelluric synthetic models of the deep sedimentary basin beneath the Institute Ice Stream in West Antarctica by way of illustration. Finally, we propose a simple empirical model by which heat exchanges between subglacial sedimentary basins and the overlying ice sheet can be estimated to first-order from electromagnetic data.

\section{Introduction}

Reconstructions of former ice sheets commonly include interactions between subglacial hydrological systems at the ice-bed interface and deeper groundwater systems below, which typically consist of several aquifer layers separated by aquitards (Boulton et al., 1995; Person et al., 2007; Piotrowski et al., 2009). Changes in ice sheet extent affect the patterns of groundwater flow, to the extent that groundwater flow directions can reverse as the ice sheet evolves. Indeed, models generally agree that, during icesheet advance and retreat, groundwater aquifers are respectively recharged and depleted (Person et al., 2007). This can introduce time lags of thousands of years between groundwater forcing and ice sheet response, and vice versa, which is highly significant for ice-sheet dynamics over glacial timescales (Lemieux et al., 2008). For example, upward groundwater flow into the subglacial hydrological system can maintain fast ice stream flow, while a reversal of flow direction can transport water from this system into the groundwater aquifer and thus cause basal freezing, so that ice stream flow switches off (Christoffersen and Tulaczyk, 2003). On catchment scales groundwater flow patterns are intimately coupled to channels in subglacial hydrological systems, with important implications for basal lubrication of ice sheet flow (Boulton et al., 2009).

Unsurprisingly, therefore, numerical models also simulate interactions between subglacial hydrological systems and deeper groundwater aquifers beneath contemporary ice sheets. This includes, for example, the Vatnajökull ice cap, Iceland (Flowers et al., 2005), or upward groundwater flow from deeper aquifers into subglacial hydrological systems beneath both the West Antarctic (Christoffersen et al., 2014) and the East Antarctic (Gooch et al., 2016) ice sheets. Up to half of all water available for basal lubrication of ice stream flow at the Siple Coast, West Antarctica, may come from groundwater (Christoffersen et al., 2014).

In the Antarctic Dry Valleys groundwater in brine saturated sediments has been delineated recently with the SkyTEM electromagnetic system (Mikucki et al., 2015). It is therefore timely and important to measure, delineate and characterise the interactions between deeper groundwater aquifers, subglacial hydrologic systems and ice stream flow in Antarctica (Siegert et al., 2017).

\section{Field site and methods}

Based on extensive airborne geophysical data acquired in 2011/11 we propose that the Institute Ice Stream system, West Antarctica, is a particularly good target for identification and characterization of subglacial groundwater, because it:

- has a steep reverse-sloping bed and is at a physical threshold of marine ice sheet instability (Ross et al., 2012);

- has evidence for regional dynamic change as recently as 400 years ago, during which the patterns of fast ice stream flow changed and the Bungenstock Ice Rise appeared (Siegert et al., 2013), possibly aided by changes in groundwater flow patterns that switched basal melting to basal freezing (Christoffersen and Tulaczyk, 2003);

- is underlain by a kilometres-deep sedimentary basin that likely hosts a thick groundwater aquifer (Jordan et al., 2013); and

- has a smooth bed in airborne radar data, indicating the presence of a soft subglacial till layer, as well as an active subglacial hydrological system (Siegert et al., 2016).

As tested here by conceptual forward and inverse modelling for the Institute Ice Stream system, an approach that combines active-source seismic with magnetotelluric (Figures 1 and 2) measurements promises to be well suited to detect, delineate and quantify the groundwater contents of subglacial sedimentary basins and, potentially, monitor groundwater exchange with subglacial till layers (Wannamaker et al., 2004; Siegert et al., 2017; Key and 
Heat and groundwater transport beneath the Antarctic Ice Sheet

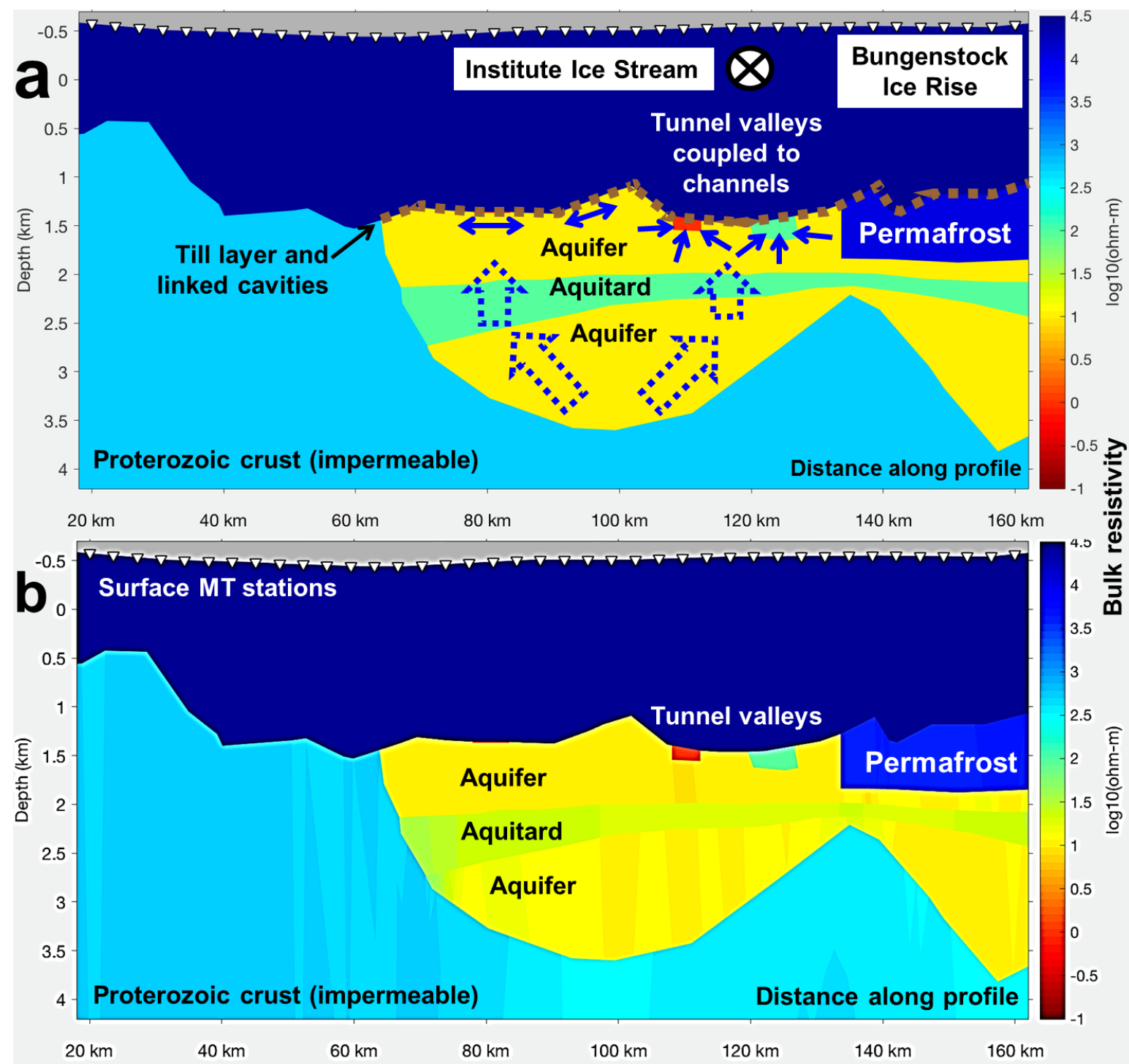

Figure 1: (a) Conceptual model for magnetotelluric feasibility study, conceptualised from existing geological interpretation of coastal profile beneath the Institute Ice Stream, West Antarctica (Fig. 5 Jordan et al. (2013)) using a three-bulk layer groundwater reservoir (Boulton et al., 1995) for illustration. A-priori model of hypothesized groundwater interactions (blue arrows) with the interfacial hydrological system between the ice sheet and the substrate, including a subglacial till layer, linked cavities, channels and subglacial lakes, including a hypothesized layer of permafrost beneath Bungenstock Ice Rise. (b) Magnetotelluric electrical resistivity tomography image of synthetic data, acquired at white triangles, with structural boundaries constrained by seismic data, showing that boundary constrained magnetotelluric inversion can recover the conductivity of subglacial aquifers, aquitards, permafrost, tunnel valleys and lakes. The till layer and linked cavities were not included in the inversion.

Siegfried, 2017). Our synthetic models for the Institute Ice Stream system (Figures 1 and 2) were generated using MARE2DEM, a freely available, parallel-adaptive, finiteelement modeling code (Key and Ovall, 2011; Key, 2016).

\section{Subglacial heat flux from magnetotelluric data?}

Electromagnetic techniques, such as magnetotellurics, hold great promise in the study of ice sheets and glaciers and their substrates (Kulessa, 2007). The magnetotelluric method infers the bulk electrical resistivity structure of the 


\section{Heat and groundwater transport beneath the Antarctic Ice Sheet}

subsurface. In a porous medium saturated with groundwater or glacial meltwaters, electrical current is typically conducted by: (i) mobile ions that flow through the pore space (electrolyte conductivity), so that bulk resistivity scales inversely with porosity and directly with fluid electrical resistivity according to Archie's law; and (ii) the surfaces of the mineral grains that make up the medium, which is known as surface conductivity and depends most strongly on clay mineral content (Archie, 1942; Kulessa et al., 2006; Kulessa, 2007; Thompson et al., 2012, 2017; Revil, 2013).

Because temperature enhances the mobility of the ions both within the pore space and at the grain surfaces in media such as unfrozen subglacial sediments, electrolyte and surface conductivities scale directly and bulk resistivity inversely with temperature. Indeed, in saturated low enthalpy media (validated for subsurface temperatures below $\sim 200^{\circ} \mathrm{C}$ ) both electrolyte and surface conductivities depend approximately linearly on temperature (Hayley et al., 2007; Hermans et al., 2014). For example, borehole studies have shown that temperature changes as small as $2{ }^{\circ} \mathrm{C}$ can be detected with electrical resistivity tomography in the near surface (Hermans et al., 2015).

Hermans et al. (2014) showed that the absolute temperature $T_{2}$ can be deduced from the initial temperature $T_{1}$ using:

$$
T_{2}=\frac{\rho_{1}}{\rho_{2}}\left(T_{1}-T_{r e f}\right)+\frac{\frac{\rho_{1}}{\rho_{2}}-1}{m_{r e f}}+T_{r e f}
$$

where $\rho_{1}$ and $\rho_{2}$ are the corresponding bulk resistivities and $m \approx 0.0187{ }^{\circ} \mathrm{C}^{-1}$ characterises the linear temperature dependence given a reference temperature $T_{r e f}=25{ }^{\circ} \mathrm{C}$ (Hayley et al., 2007). Although applied in an electrical resistivity tomography monitoring study in Hermans et al. (2014), equation (1) can equally be applied in a spatial sense where heat flow estimation is of concern.

Previous in-situ low-frequency electrical measurements of unfrozen subglacial sediments (Blake and Clarke, 1999; Kulessa et al., 2003a, 2006, in press; Siegert et al., 2017), subglacial sedimentary rock (Wannamaker et al., 2004) and solid earth tide forcing of meltwater flow in subglacial sediments (Kulessa et al., 2003b) lead us to expect that temperature will affect ionic mobility in their pore spaces and at their grain surfaces. We can therefore apply equation (1) to the sedimentary basin beneath the Institute Ice Stream to demonstrate that sensible values of heat fluxes are derived given temperature estimates.

Fisher et al. (2015) applied Fourier's law to calculate the heat flow $(q)$ beneath Lake Whillans at Antarctica's Siple Coast:

$$
q=-\lambda \frac{T_{2}-T_{1}}{d_{2}-d_{1}}
$$

where $\lambda$ is the thermal conductivity $(\sim 2.8 \mathrm{~W} /(\mathrm{m} \mathrm{K})$ for sandstone (Gooch et al., 2016)), and $d$ is the thickness of the sedimentary basin if the elevation of the ice base is set as $d$ $=0$. Here we use T base $=50^{\circ} \mathrm{C}$, as calculated for a depth of $\sim$ $2 \mathrm{~km}$ below the ice base in a major sedimentary basin in East Antarctica (Gooch, 2016). We can then:

1. Apply equation (1) to calculate the temperature at the top of the sedimentary basin $\left(\mathrm{T}_{\mathrm{top}}\right)$ - equal to the elevation of the ice sheet base - in dependence of the resistivity ratio between the base ( $\left.\rho_{\text {base }}\right)$ and top $\left(\rho_{\text {top }}\right)$ of the sedimentary basin. For an ice base at the pressure melting point ( $\mathrm{T}_{\text {top }}$, approx. $-2{ }^{\circ} \mathrm{C}$ ) the corresponding resistivity ratio is $\sim 0.35$ (Figure 3 ) (i.e. we would expect to measure a three-fold increase in the bulk resistivity of the sedimentary basin from the base to the top if other key properties do not change).
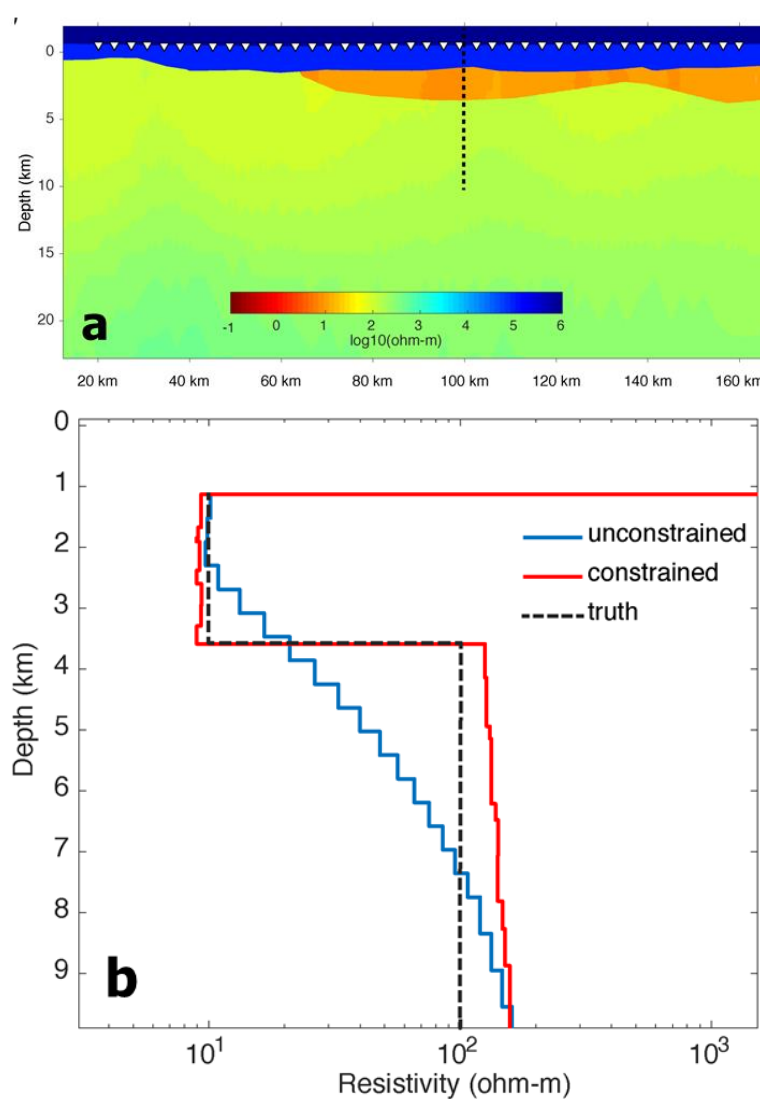

Figure 2: Comparison of unconstrained versus constrained magnetotelluric inversion for the same subglacial groundwater basin shown in Figure 1. 


\section{Heat and groundwater transport beneath the Antarctic Ice Sheet}

2. Apply equation (2) to calculate the corresponding heat fluxes between the base and the top of the sedimentary basin in dependence of the calculated temperature (or bulk resistivity ratio) changes. In this case we get $\sim 70 \mathrm{~mW} / \mathrm{m}^{2}$ for the resistivity ratio of $\sim 0.35$.

\section{Conclusions}

We have argued that coupled groundwater and heat fluxes are highly likely to impact ice sheet dynamics and evolution on centennial and millennial timescales. We have shown that electromagnetic techniques such as magnetotelluric surveys show considerable promise in delineating groundwater reservoirs and their coupling with subglacial hydrological systems, and may also facilitate estimations of geothermal heat fluxes into the ice sheet base. Our feasibility study is simplistic in that it negates likely changes in subglacial sedimentary basin properties such as lithology, porosity or water saturation or salinity. McIntosh et al. (2011) show for example that the Michigan sedimentary basin experiences approximately a four-fold increase in groundwater salinity from the surface to a depth of $\sim 2 \mathrm{~km}$. Other available evidence, e.g. from seismic, gravity or magnetic techniques with broad glaciological track records or indeed up and coming glacier seismoelectric surveys (Kulessa et al., 2006, in press; Siegert et al., 2017; Monachesi et al., 2018) and / or hydrological modelling, should therefore be integrated with future magnetotelluric interpretations.

\section{Acknowledgments}

We acknowledge financial support from UK Natural Environment Research Council Grants NE/S006621/1, NE/R010838/1, NE/G013071/1.
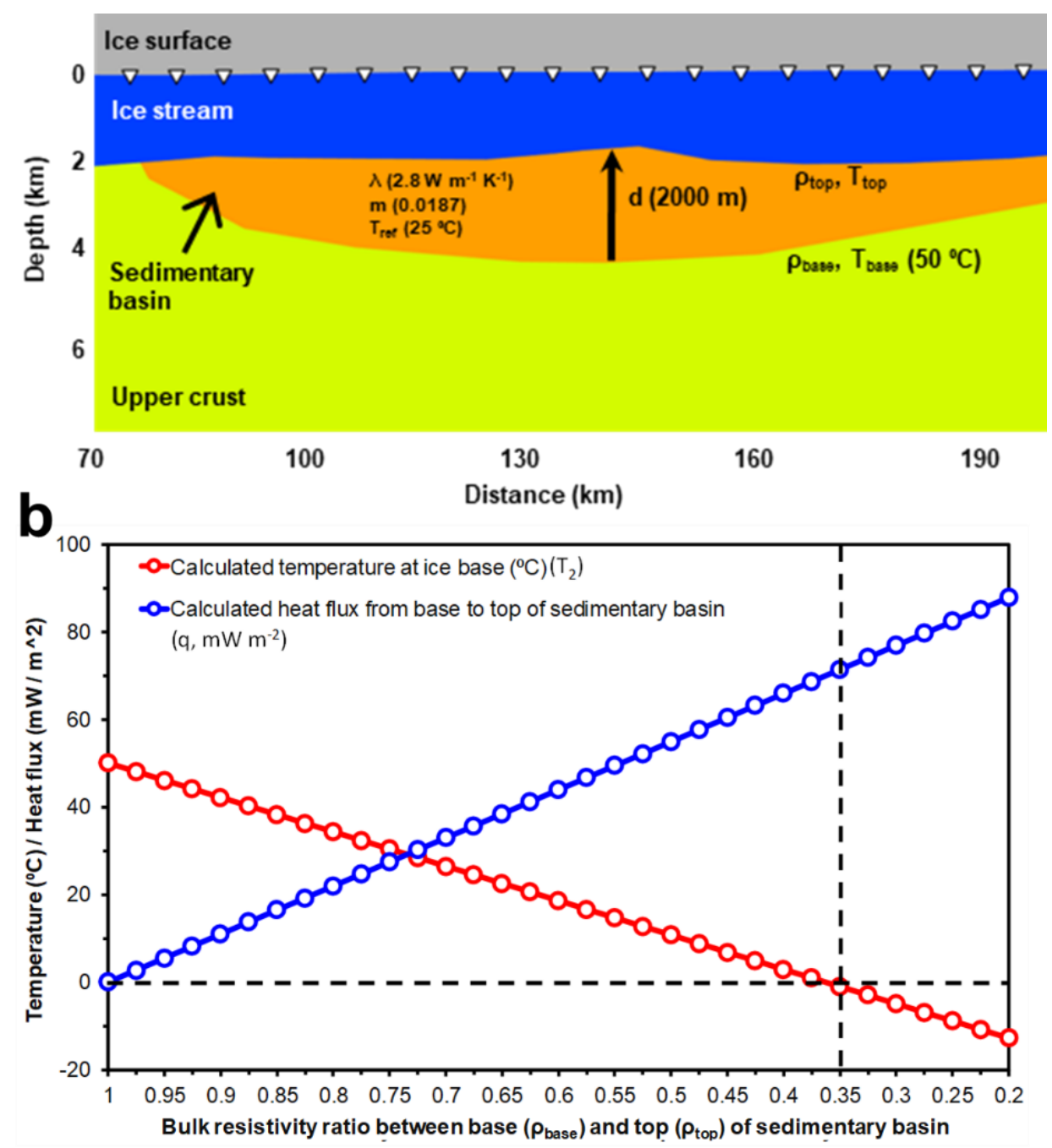

Figure 3: (a) Model setup used for (b) illustrative calculation of geothermal heat fluxes from the base to the top of the sedimentary basin from bulk resistivity ratios, as well as associated simulated temperature at the ice sheet base. 